\title{
Comercialización, consumo y valor nutricional de la comida rápida, en los estudiantes universitarios, caso: carrera de agroindustria de la facultad de ciencias pecuarias
}

\section{Marketing, consumption and nutritional value of rapid food, in university students, case: career of agroindustries of the faculty of animal ground sciences}

\author{
María Belén Bravo Avalos", José Luis López Salazar², Luis Antonio Velasco \\ Matveev¹, and María Fernanda Miranda1 \\ ${ }^{1}$ Carrera de Agroindustria, Facultad de Ciencias Pecuarias, Escuela Superior Politécnica de \\ Chimborazo \\ ${ }^{2}$ Carrera de Administración de Empresas, Facultad de Administración de Empresas, Escuela \\ Superior Politécnica de Chimborazo
}

Received: 10 January 2020

Accepted: 17 January 2020

Published: 26 January 2020

Publishing services provided by

Knowledge E

(c) María Belén Bravo Avalos et al. This article is distributed under the terms of the

Commons Attribution License, which permits unrestricted use and redistribution provided that the original author and source are credited.

Selection and Peer-review under the responsibility of the $\mathrm{VI}$ Congreso Internacional Sectei 2019 Conference Committee.

\section{Resumen}

Se considera comida rápida a toda clase de comida preparada de manera rápida, cómoda y por lo general barata y de amplia comercialización. El presente artículo contribuye con definiciones, características, ventajas y desventajas del producto, así como sus consecuencias en la salud en las personas. El objetivo fue identificar cual es la comida rápida de mayor consumo y a partir de este obtuvo el valor nutricional de la comida rápida que resultó consumida en mayor cantidad, así como también se conocieron cuáles son los factores que influyen en su consumo. La metodología que se empleó fue el enfoque cuantitativo y cualitativo, se aplicó encuestas a los estudiantes de Facultad de Ciencias Pecuarias de la Escuela Superior Politécnica de Chimborazo, periodo académico abril -- agosto 2018, también se analizó los productos de mayor consumo en el laboratorio de la Facultad de Ciencias Pecuarias. El resultado obtenido demostró un alto porcentaje de estudiantes que consumen comida rápida a pesar de estar conscientes que es de mala calidad y que afecta a la salud.

Abstract: It is considered fast food to all kinds of food prepared in a fast, comfortable way and in general cheap and of extensive marketing. The present article contributes with definitions, characteristics, advantages and disadvantages of this product, such as its consequences related to people's health. The aim was to identify which is the most consumed fast food and obtained its nutritional value that turns out to be mainly eaten, as well as knowing which are the factors that influence on its consumption. The methodology used is the quantitative and qualitative approach, surveys were applied to 108 students of the Career of Agroindustries of the Escuela Superior Politécnica de Chimborazo, during the academic period April - August, 2018, also the most consumed food were analyzed in the laboratory of the Animal Ground Sciences Faculty. Obtained 
results demonstrate a high percentage of students who consume fast food in spite of being conscious about its poor quality and health issues.

Palabras claves: Comercialización, comida rápida, valor nutricional, impacto.

Keywords: Marketing, fast food, nutritional value, impact.

\section{Introducción}

\subsection{Antecedentes del consuno y comercialización de la comida ráp- ida}

En 1940 en Estados Unidos se hace muy popular servir comidas sin necesidad de salir de un coche, en New York se abre el primer automat, un local que ofrecía comida detrás de una ventana de vidrio y una ranura para pagar con monedas. El sistema ya existía antes en Berlín y en algunas ciudades de Estados Unidos como Filadelfia. La firma popularizó la comida para llevar bajo el eslogan "menos trabajo para mamá" (1).

Según Nathional Geography la comida rápida empezó en 1948 cuando dos hermanos de apellido McDonald's (Rick y Mac) introdujeron un restaurant denominado "drive-in" que consistía en llevar la comida a los autos en las afueras de este, pero no se quedaron conformes ya que con el paso de los años innovaron su negocio, se introdujo un sistema de producción en cadena, Ilamado "Speedee Service System", de manera que los empleados sólo tuvieran que intervenir en contadas ocasiones en el producto final (2). Con estos antecedentes comienza a hacerse cada vez más popular y de fácil acceso para los consumidores, por lo tanto, a incrementar su comercialización.

Se considera comida rápida a toda clase de comida preparada de manera rápida, cómoda y por lo general barata (3). La comida rápida se refiere a un establecimiento donde primero se paga el consumo antes de que se sirva y donde no hay meseros. Sin embargo, las modalidades de servicio y de alimento de la comida rápida se pueden dividir en varias categorías: Para llevar, en el mismo restaurante o a domicilio (4).

La comida rápida ha sido y sigue siendo un éxito internacional, avanzando a países subdesarrollados ampliando masivamente la existencia de los fast food. Su triunfo radica en la rapidez del servicio, y por otro lado en los bajos precios y la flexibilidad de horarios. Estas son las razones que llevan a las personas a frecuentar los establecimientos de comida rápida: la falta de tiempo y de poder monetario, además de que a casi cualquier hora del día se encuentran abiertos (5). 
La comida chatarra es una denominación del argot para los alimentos con valor nutricional limitado, por lo general, ofrece poco en términos de proteínas, vitaminas o minerales en cambio aportan una gran cantidad de calorías de azúcar o grasa, lo que se ha denominado con el término "calorías vacías". Considera que los fritos son algunos de los principales alimentos considerados como comida chatarra (6). No se usa masivamente el término chatarra para evitar su disminución de consumo, por lo que la palabra más utilizada es comida rápida, construyendo una imagen positiva y atractiva hacia los consumidores.

Esta comida rápida va dirigida a toda la población, pero sobre todo a la población con un ritmo de vida acelerado, que manejan rangos de tiempo muy cortos para el consumo de alimentos, y es así que debido al ritmo de vida que llevan estudiantes universitarios, es poco común que se coma en casa y mucho menos se preparen los alimentos de manera apropiada. Una de las razones del porqué la comida chatarra es tan popular es debido a su bajo costo. Es decir, uno puede ir a cualquier restaurante de comida rápida y ordenar, con poco dinero, un menú que saciará el hambre por un par de horas. Contrariamente, los alimentos saludables, como las frutas frescas y vegetales, tienden a ser más costosas (7).

Los productos de fast food se pueden clasificar en dos grupos: los de influencia y características anglosajonas, como hamburguesas, salchichas y patatas fritas acompañadas de salsas diversas (mayonesa, mostaza, ketchup...), y los de procedencia mediterránea, como pizzas, bocadillos, tapas y kebabs (pinchos de carne adobada de origen árabe envueltos en pan de pita y acompañados de vegetales y salsas de yogur). Por sus características nutricionales, la comida rápida de estilo mediterráneo o árabe se puede considerar más saludable que la de estilo sajón (8).

Ventajas de la comida rápida:

- Entre las mayores ventajas que aporta el consumo de comida rápida está el ahorro de tiempo, ya que al comer en un restaurante fast food no se tiene que cocinar, además muchos de estos restaurantes ofrecen la posibilidad de llevar la comida al propio domicilio, por lo que la comodidad se incrementa (9).

- Aporta el consumo de comida rápida está el ahorro de tiempo, ya que al comer en un restaurante fast food no se tiene que cocinar; además muchos de estos restaurantes ofrecen la posibilidad de llevar la comida al propio domicilio, por lo que la comodidad se incrementa (10).

- Por lo general tienen buen sabor, son baratas, se pueden comer estando de pie, no hay que lavar platos (11). 
- Poseen alto contenido de calorías que dan energía al cuerpo. Además, provee hidratos de carbono, proteínas, grasas, vitaminas y minerales (12).

Desventajas de la comida rápida:

- La energía proviene de grasas saturadas y azúcares que, en exceso, son perjudiciales para la salud y carecen de otros nutrientes esenciales (13).

- Un estudio publicado en 2012 en "The American Journal of Clinical Nutrition" encontró que el consumo regular de comida chatarra de alta densidad energética disminuye la saciedad sensorial específica, haciendo que comas más de ese alimento. Si comes demasiado consistentemente, especialmente comida chatarra, esto puede conducir al aumento de peso y la obesidad (14).

La comercialización de estos productos es un factor que contribuye al sobrepeso y obesidad, pero son los medios de comunicación quienes juegan un papel importante en la percepción y aceptación de diferentes comidas rápidas con un atractivo que llama la atención, estas se presentan a la población y siguiendo con algunas promociones que cautivan a todos los sectores a niños, adolescentes, jóvenes, adultos, logrando su adquisición, sin necesidad que estén en su consumo habitual.

Actualmente el sector estudiantil, representado por los jóvenes, son el grupo de mayor riesgo a sufrir enfermedades transmisibles, infecciones por la inadecuada manipulación, o no poca cocción o no cocción a término. Entre las principales se conoce a nivel mundial el consumo de comidas como hamburguesas, salchipapas, papas fritas, pollo frito, chocolates golosinas, pizza, entre otras, son de fácil consumo por su portabilidad. Y se está creando un hábito, favoreciendo al incremento de peso en base a un aumento de reserva energética con altas consecuencias en jóvenes y las consecuencias incluso académicas (15).

Consecuencias para la salud por el consumo de comida rápida:

Según la nutricionista Francisca Cifuentes, explica que el consumo de este tipo de alimentos está relacionado con enfermedades como el sobrepeso, obesidad, diabetes, problemas cardiovasculares, presión alta y también se elevan los niveles de colesterol y triglicéridos; además manifiesta que este consumo es más visible en el periodo de la universidad ya que la falta de tiempo y un cambio en los hábitos alimenticios hace que las personas opten por consumir estos alimentos (16).

Además, se conocen también problemas como:

- Enfermedades del corazón: Es una de las afecciones más comunes a causa del consumo de comida rápida. Grandes cantidades de grasa corporal suelen dar 
lugar a mayores niveles de colesterol LDL y niveles bajos de HDL. Ambos factores pueden aumentar el riesgo de enfermedades cardiacas, una de las principales causas de muerte en el mundo (17).

- Problemas digestivos: Causa el reflujo o síndrome de colon irritable, ya que este tipo de alimentos se fríen. El aceite en la comida se deposita en las paredes del estómago y aumenta la producción de ácido, lo que irrita el estómago y agrava el reflujo y la digestión (18).

- Muerte: Investigadores de la School of Public Health encontraron que las personas que consumen comida chatarra, incluso una vez a la semana, aumenta $20 \%$ el riesgo de morir por enfermedades coronarias (19).

La última Encuesta Nacional de la Situación Nutricional realizado en Colombia, elaborada por la Organización Mundial de la Salud en 2010, uno de cada tres jóvenes, entre 18 y 24 años, no come frutas diariamente y 5 de cada 7, no consume verduras. Por otra parte, el estudio realizado por la Pontificia Universidad Católica de Valparaíso, en Chile, analiza la relación de los hábitos alimenticios, la actividad física y el nivel socioeconómico en estudiantes universitarios, demostrando que esta población es vulnerable a una mala nutrición porque no consume merienda, no desayuna, ayuna por largas horas, prefiere las comidas rápidas y no realiza ningún tipo de actividad física (20).

Una investigación de estudiantes de Medicina del Instituto Tecnológico de Santo Domingo (INTEC) reveló que $65 \%$ de los jóvenes que realizan estudios universitarios consume comida rápida de manera excesiva. El complejo horario de clases de los jóvenes universitarios es una de las principales razones que los lleva a consumir comidas rápidas; de acuerdo a los resultados, un $65 \%$ de los estudiantes se encontraba en sobrepeso según su índice de Masa Corporal; y solamente un 20\% estaba dentro de los valores óptimos recomendables según la Organización Mundial de la Salud (OMS). Sobre la frecuencia en el consumo de estos alimentos, la investigación arrojó que 19 de los consultados, un 35\% de la muestra, consume comida rápida entre 1 y 2 veces por semana; seguidos por un 30\%, es decir 18 casos, que consume este tipo de comida 3 ó 4 veces por semana, luego un 16.67\% que equivale a 10 estudiantes, quienes dijeron consumir comida rápida alrededor de 5 a 6 veces a la semana y entre los que consumen comida rápida más de 6 veces por semana se encuentran 11 estudiantes, lo cual equivale a un $18.33 \%$ (21). 


\section{Metodología}

Esta investigación de diagnóstico utilizó un método inductivo-deductivo y semi experimental, los datos obtenidos se sometieron a un análisis descriptivo donde se contempló medidas de tendencia central y de dispersión; se analizó variables como: Componente social, comercialización, nivel de consumo y valor nutricional de la comida rápida.

\subsection{Descripción y contexto de los participantes}

La encuesta planteada se aplicó en una universidad de la provincia de Chimborazo, a los estudiantes de la Facultad de Ciencias Pecuarias, a 800 estudiantes como caso práctico de estudio como base hacia las demás universidades interesadas en este tipo de estudio.

\subsection{Instrumento:}

El instrumento aplicado fue el cuestionario estructurado con 12 preguntas cerradas. En el laboratorio para determinar los componentes de la comida rápida más consumida (papas fritas) se utilizó materiales como vaso de precipitación, bandejas, crisol, capuchón de celulosa, estufa de secador, mufla y máquina extractora de grasa para las papas fritas.

\subsection{Procedimiento:}

La encuesta se realizó de una forma denominada "por bloques", es decir, a quienes se les aplicará la encuesta serán los estudiantes de la Facultad de Ciencias Pecuarias.

Se planificó aplicar en la jornada matutina a los estudiantes de tercero a noveno semestre y en la jornada vespertina a los estudiantes de primer y segundo semestre.

\section{Desarrollo y Discusión}

En la investigación se determinó que el 53.7\% de la población objeto de estudio, consume en mayor cantidad comida rápida, siendo este porcentaje representado por los hombres de la Facultad de Ciencias Pecuarias, mientras que las mujeres representan el $46,3 \%$; se determinó que el $75.92 \%$ de los encuestados comprenden el rango de 
edad de 18 a 20 años, siendo el $74.07 \%$ de la población total encuestada quienes consumen comida rápida.

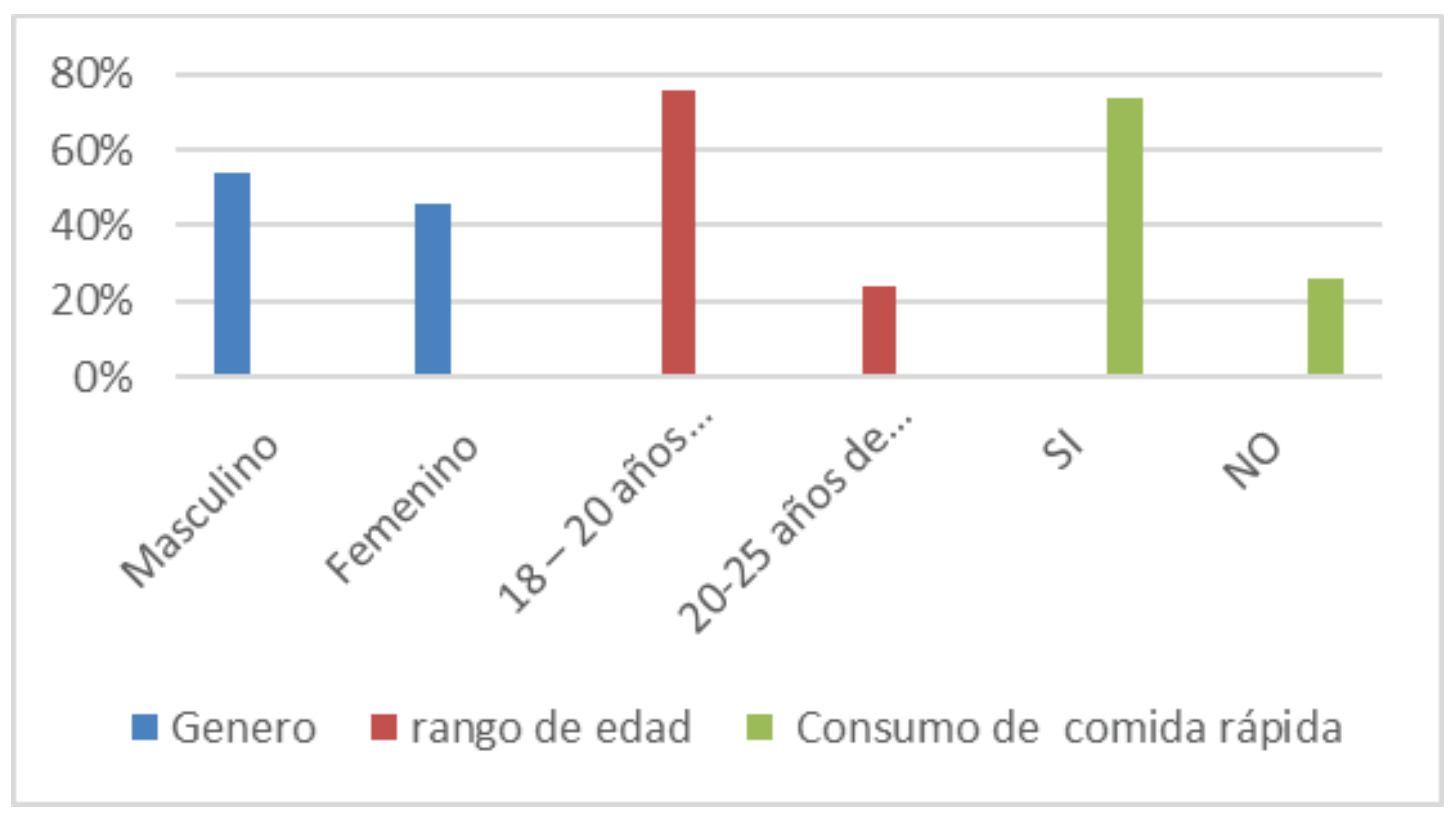

Figure 1: Tipos de consumidores.

La investigación nos permitió conocer que el $45.37 \%$ de la población consumen rara vez esta comida, pero también el 35.18\% la consumen usualmente. Se obtuvo como resultado que el alimento consumido en mayor cantidad por los estudiantes son las papas fritas con un $60.18 \%$, observando que las razones principales por las que se consumen esta comida son por falta de tiempo (77.7\%) y por su sabor (34.25\%).

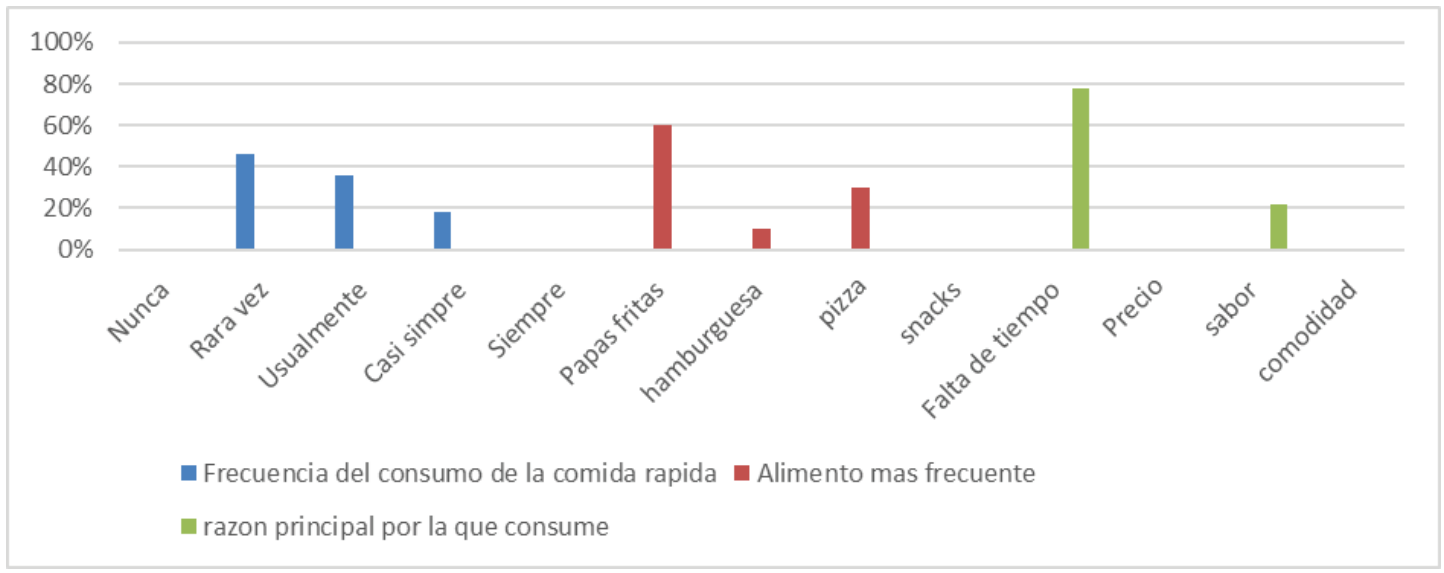

Figure 2: Nivel de consumo.

El 60.18\% hace de la comida rápida su almuerzo, adquiriendo la mayoría en puestos de comida (65.74\%). El 66.66\% de los estudiantes consideran que no hay las adecuadas normas de higiene al momento de preparar esta comida. 


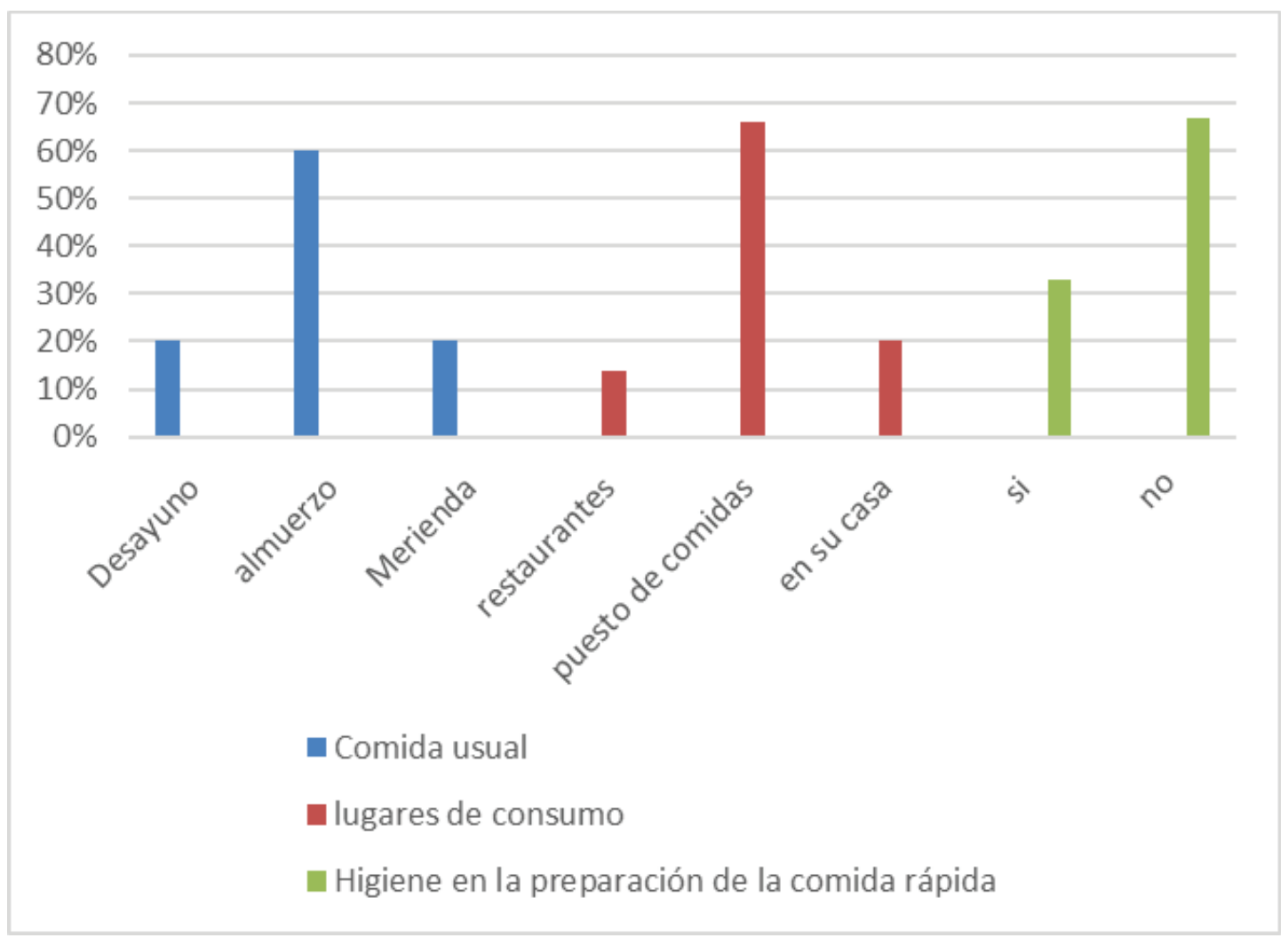

Figure 3: Preferencia de comida rápida.

Del total encuestados, el $85.59 \%$ concuerda en que la comida rápida es mala porque afecta a la salud en la actualidad como a futuro, esto debido a sus componentes, que no son nutritivos y por la inadecuada higiene que se mantiene al momento de la transformación en productos listos para su consumo.

Además, se obtuvo que el 51.85\% de los encuestados sí ha recibido información sobre el consumo de comida rápida, mientras que el $48.15 \%$ no ha recibido información, detalle importante para conocer el consumo y las razones, así como la comercialización que realizan y no pecar de ignorancia, el nivel de conocimiento ayuda a saber las estrategias de comercialización.

Finalmente se determinó que el 39.81\% acepta gradualmente dejar de consumir comida rápida y reemplazarla por alimentos más nutritivos, mientras que el 11.1\% definitivamente no dejará de consumir comida rápida, a expensas del conocimiento total de las grandes desventajas y pocas ventajas que tienen el consumo de comida rápida, repercutidas en el corto y largo plazo sobre la salud de las personas, la economía y los factores sociales que influyen al realizar estas actividades de consumo.

Luego de haber realizado la encuesta se concuerda que la falta de tiempo es una de las razones por la que los estudiantes acuden a consumir este tipo de comida en diferentes lugares (5). Así como también a que debido al ritmo de vida que llevan los 


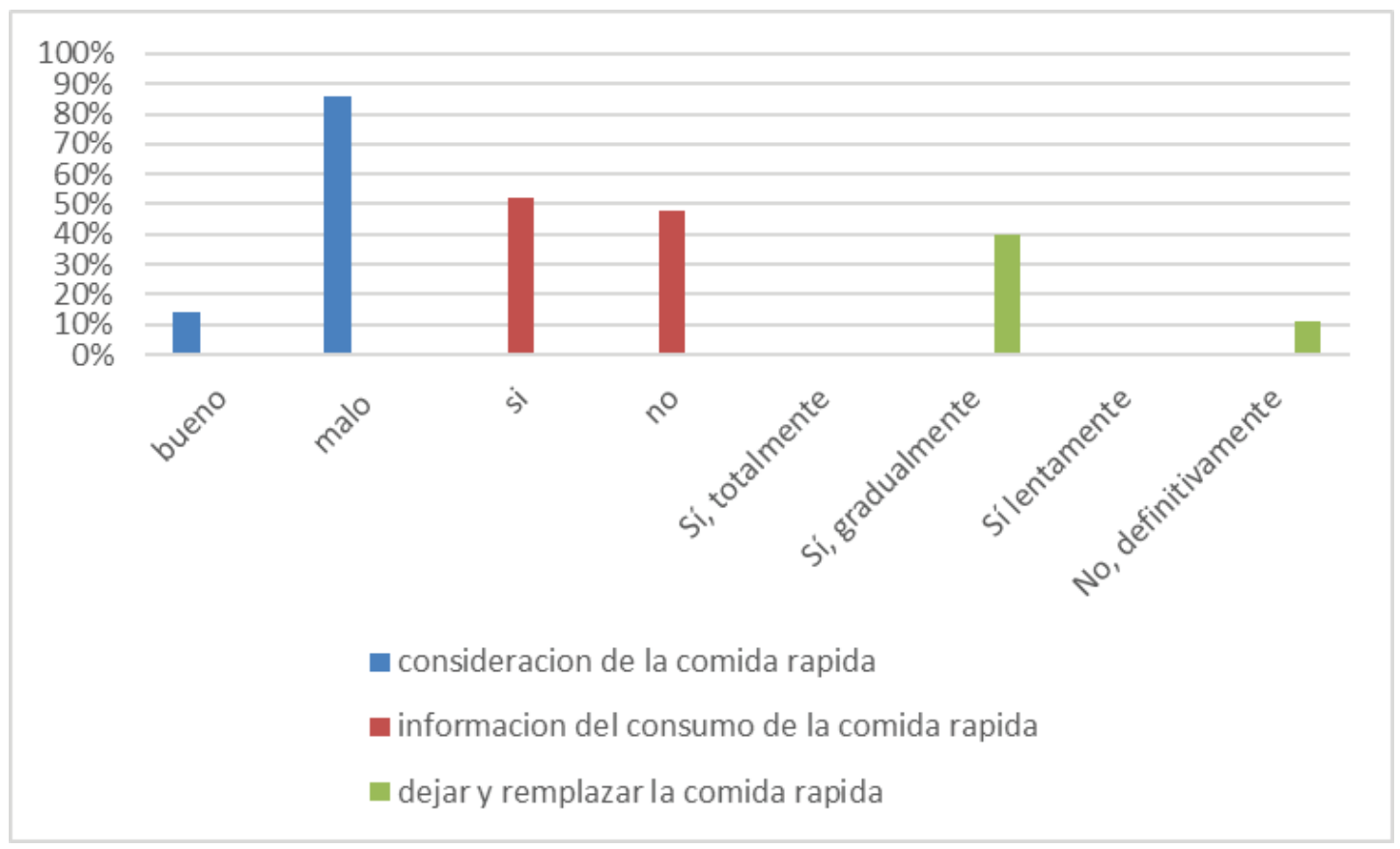

Figure 4: Comercialización de comida rápida.

estudiantes universitarios, es poco común que se coma en casa y mucho menos que se preparen los alimentos de manera adecuada (7).

Para determinar el grado de valor nutricional y contenido de grasa, humedad, carbohidratos y proteínas se analizó una muestra en el Laboratorio de Alimentos de la Facultad de Ciencias Pecuarias. Obteniendo los siguientes resultados: En 100 gramo de papas fritas hay un $29,02 \%$ de grasa, humedad de $52,89 \%$, carbohidratos $14,75 \%$ y proteínas apenas un 3,34\%.

Se recalca que los consumos excesivos de este tipo de alimentos bajos en propiedades nutritivas provocan obesidad y enfermedades perjudiciales para la salud, esta investigación permite afirmar que la comida rápida para los estudiantes es la salida más rápida, fácil por su gran nivel de comercialización para saciar el hambre, la población investigada está consciente del daño que puede causar, pero a pesar de ello opta por consumirla; ante esto los investigadores concordamos con la investigadora antes citada al manifestar que consumimos este tipo de comida por falta de tiempo y además ocasiona secuelas en la salud(16).

La comida rapida no aporta con un valor nutritivo adecuado para nuestra salud por lo que debemos cambiar nuestros habitos alimenticios, son tan ciertos y comprobados en esta investigación sobretodo en el nivel universitario que la comercialización de comida rápida es mas amplia y fácil de adquirirla por su cercanía y costo económico (14). Consideran que el desorden alimenticio de los jóvenes universitarios es elevado 


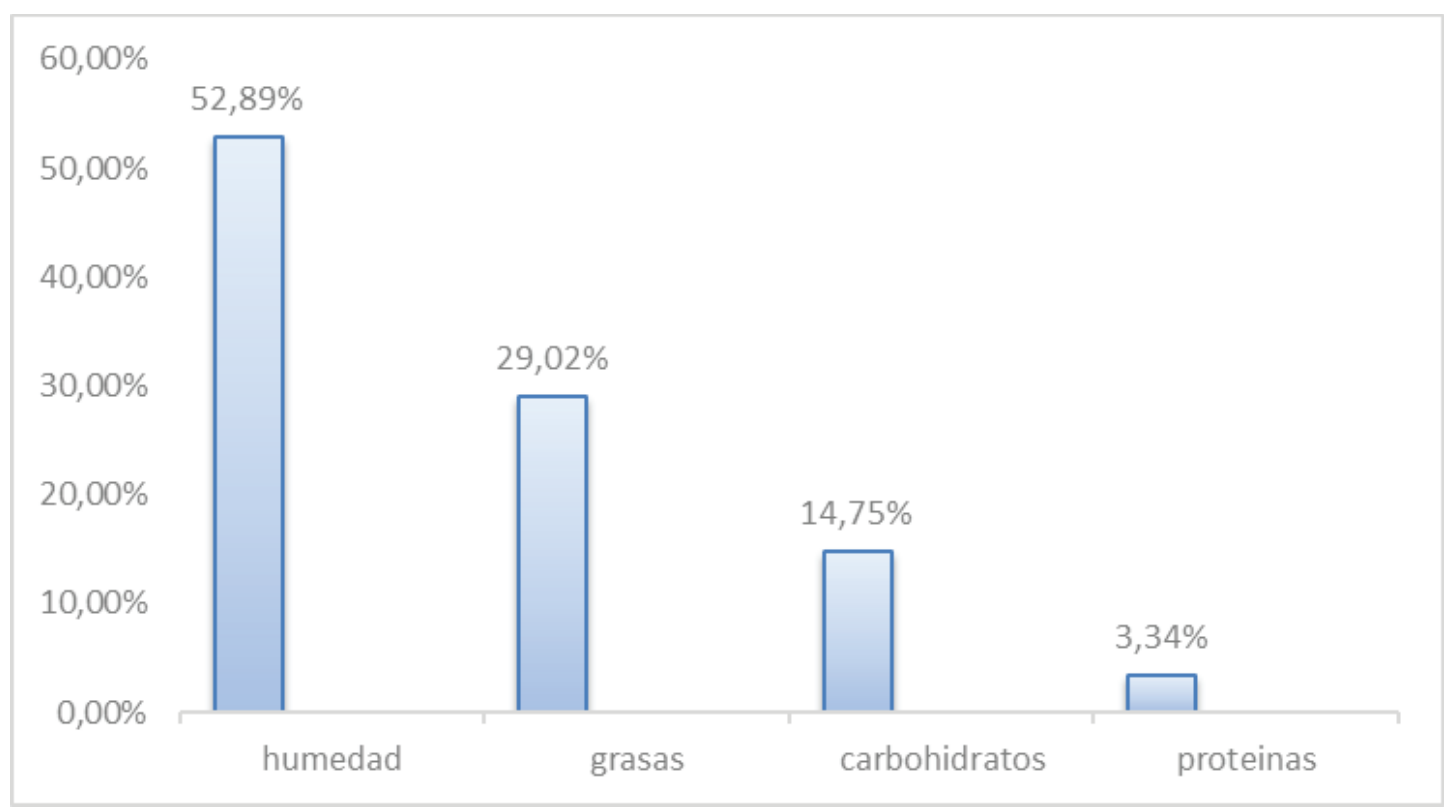

Figure 5: Valor nutricional de las papas fritas.

por el poco tiempo que disponen y esto los conlleva a consumir comida rápida de manera excesiva, exponiéndolos a enfermedades cardiovasculares, diabetes, y sobre todo la obesidad dando certeza a la investigación realizada en una carrera de tercer nivel de una universidad pública como se ha presentado en esta investigación (21).

Los peligros causados por la comida rápida en la salud a corto, mediano y largo plazo, ya que las enfermedades del corazón son uno de los problemas principales que causa la muerte en el mundo, la grasa aumenta el colesterol malo y como consecuencia causa enfermedades cardiacas, impactantes y fulminantes en la vida de los seres humanos (17).

La pronta asimilación de ácidos grasos al hígado procedentes de la grasa, va implicando mayor riesgo cardiovascular en los consumidores de estos productos, estos síntomas junto con la resistencia a la insulina y la obesidad se favorecen por el creciente sedentarismo en la población actual. Más de dos tercios de los pacientes con enfermedad coronaria tienen sobrepeso u obesidad. Uno de los factores que más contribuyen al aumento de los casos de diabetes son las dietas poco saludables, mientras que dietas saludables proveen al cuerpo un flujo constante de glucosa, ayuda a mantener la sensibilidad a la insulina. Al consumir en exceso la comida rápida o chatarra, puede existir deficiencias en los micronutrientes tales como las vitaminas y minerales, puesto que los alimentos de alta densidad energética o mayor densidad carecen con frecuencia de proteínas, vitaminas como la A, C, D,E y el complejo B, calcio, hierro potasio, zinc y grasas insaturadas. Una deficiencia en cualquiera de estos nutrientes compromete al organismo y sistema inmunológico, de la misma manera no 
habrá una adecuada producción de serotonina, en consecuencia, ansiedad, depresión e irritabilidad (22).

El nivel de consumo versus las enfermedades quedan relegados por la facilidad de adquisición que se tiene de estos productos y la escaza oferta de productos saludables en los alrededores de la población objeto de estudio, además el bajo y competitivo costo frente a productos más saludables, pero sobretodo la gran publicidad que llega frecuentemente a la población joven; con todos estos detalles, se facilita su adquisición, es por eso que el mercado de la famosa comida rápida ha crecido notablemente, sus consumidores se han ido sumando hasta abarcar un número amplio de mercado.

\section{Conclusiones}

- Existe un alto consumo de comida rápida, esto se produce debido al actual estilo de vida de la población y el poco tiempo que mantienen tanto para la preparación, como para el consumo de alimentos, incrementando su comercialización, hecho observado principalmente en estudiantes universitarios, como el caso investigado en los estudiantes de la Facultad de la Ciencias Pecuarias, en donde se presenta un $53,7 \%$ de consumo de este tipo de comida.

- La comida rápida conocida también como chatarra posee un bajo o nulo aporte nutricional que mantienen este tipo de productos, pero que son consumidos porque producen satisfacción sensorial. Al analizar el principal producto consumido por los estudiantes en donde se aplicó la investigación se obtuvo las papas fritas, mismas que al realizar un análisis nutricional se comprueba el bajo aporte que tienen para el cuerpo humano, con apenas el 3,34\% de proteínas.

- La satisfacción que producen al ser consumido, la fácil adquisición de estos productos y su fácil transportación debido a sus envases, permiten que el consumo siga en línea ascendente; la publicidad visual y boca a boca hacen que estos productos sean adquiridos en mayor cantidad por los estudiantes incrementando constantemente la demanda.

\section{References}

[1] Fajardo, E. (s.f). Comida rápida. Recuperado el 20 de 05 de 2018, de calaméo: https://es.calameo.com/read/005077506d78f5d4b7e9a

[2] National Geographic. (4 de 10 de 2015). Historia de la comida rápida. Obtenido de National Geographic: http://www.ngenespanol.com/el-mundo/hoy/15/04/9/asi- 
inicio-la-comidarapida/

[3] Guías de la Salud. (16 de Junio de 2009). Información sobre comida rápida. Recuperado el 05 de Mayo de 2018, de youngwomenshealth.org: https:// youngwomenshealth.org/2009/06/16/comida-rapida/

[4] Club planeta. (s.f). La comida rápida:características y ventajas. Obtenido de trabajo.com.mx: http://www.trabajo.com.mx/ la_comida_rapida_caracteristicas_y_ventajas.htm

[5] Chávez, O., Humberto, O., \& Díaz, F. (2013). Consumo de comida rápida y obesidad, el poder de la buena alimentación en la salud. Iberoamericana para la Investigación y el Desarollo Educativo, 182.

[6] Orjuela, R. E. (marzo de 2017). Que es la comida chatarra? Obtenido de nocomasmentiras.org: http://www.nocomasmasmentiras.org/archivos/ Que_es_comida_chatarra.pdf

[7] Rodriguez, L. (18 de Diciembre de 2015). Casa Verde Gourmeth. Obtenido de https: //www.casaverdegourmet.com.pe/blog/comida-chatarra-razones-consumo/

[8] Zarraga, A. (15 de Febrero de 2004). EROSKI CONSUMER, el diario del consumidor . Obtenido de http://revista.consumer.es/web/es/20040201/alimentacion/68091.php

[9] Gomez, A. (15 de Mayo de 2003). Exite. Obtenido de http://gastronomia.excite.es/ comidas-rapidas.html

[10] García, B. M. (3 de Diciembre de 2013). La verdad . Obtenido de http: //www.miperiodicodigital.com/2014/edicion2014/ccbi/23583-la-comida-basuraventajas-desventajas.html

[11] Requena, M. (08 de Abril de 2015). Comida Chatarra. Obtenido de http:// comidaucab95.blogspot.com/2015/04/ventajas-y-desventajas-de-la-comida.html

[12] Cuyo, R. D. (30 de Marzo de 2017). Diario De Cuyo. Obtenido de https: //www.diariodecuyo.com.ar/salud/Ventajas-y-desventajas-de-la-comida-rapida20170330-0092.html

[13] Orzola, M. (03 de Abril de 2017). BigBang. Obtenido de https://www.bigbangnews. com/comunidad/Ventajas-y-desventajas-de-la-comida-rapida-20170329-0045. html

[14] Fisk, M. (10 de Mayo de 2017). muy fitness. Obtenido de https://muyfitness.com/ desventajas-comida-chatarra-info_28494/

[15] Palacios, M. (15 de Abril de 2014). CLub Planeta. Obtenido de http://www.trabajo. com.mx/la_comida_rapida_caracteristicas_y_ventajas.htm

[16] Cifuentes, F. (2 de Septiembre de 2014). ¿Qué es la comida 'chatarra'? El Comercio. 
[17] Alcumedo, V. M. (04 de julio de 2017). Los 10 peligros causados por la comida rápida. Recuperado el 19 de mayo de 2018, de El Universal De10.mx: http://archivo.de10. com.mx/8513.html

[18] Universia. (29 de agosto de 2017). 10 consecuencias de abusar de la comida basura. Recuperado el 19 de mayo de 2018, de universia.es: http://noticias.universia.es/ cultura/noticia/2015/09/28/1131651/10-consecuencias-abusar-comida-basura.html

[19] Salud180.com. (s.f.). 5 consecuencias de comer en exceso comida chatarra. Recuperado el 5 de mayo de 2018, de Salud180.com: http://www.salud180.com/ 5-consecuencias-de-comer-en-exceso-comida-chatarra

[20] ElHeraldo.co. (24 de noviembre de 2014). Universitarios: buena educación, pero mala nutrición. El Heraldo, págs. https://www.elheraldo.co/tendencias/universitariosbuena-educacion-pero-mala-nutricion-175158.

[21] Brito, J., Luciano, J., Pérez, K., \& Matos, J. (12 de Enero de 2017). Estudiantes universitarios consumen comida "chatarra" en exceso. Recuperado el 5 de mayo de 2018, de El Dia.com: http://eldia.com.do/estudiantes-universitarios-consumencomida-chatarra-en-exceso/

[22] Ruiz M, (2014). Manual de nutrición y dietética, cuadernos hospital de clínicas. Páginas 14-28. 